\title{
Skin insertion site culture for the prediction of primary bloodstream infection
}

\author{
Leonardo Lorente ${ }^{1} \oplus$ - María Lecuona ${ }^{2} \cdot$ Alejandra Pérez-Llombet ${ }^{1} \cdot$ Adriana González-Mesa $^{1} \cdot$ Manuel Callejon $^{2}$. \\ Alejandro Jiménez ${ }^{3} \cdot$ María Luisa Mora' ${ }^{1}$ Ana Madueño ${ }^{2}$
}

Received: 25 May 2021 / Accepted: 9 June 2021 / Published online: 14 June 2021

(c) Royal Academy of Medicine in Ireland 2021

\begin{abstract}
Purpose Previous studies have analyzed the capability of skin insertion site culture to predict catheter-related bloodstream infection (CRBSI). However, there has been not analyzed its capability to predict primary bloodstream infection (PBSI), that include CRBSI and bloodstream infection of unknown origin (BSIUO). The novel objective of our study was to determine the capability of insertion skin site culture to predict CRBSI and primary bloodstream infection (PBSI), that include CRBSI and bloodstream infection of unknown origin (BSIUO).

Material and methods Observational and prospective study in one Intensive Care Unit. Patients with some central venous catheter (CVC) at least during 7 days and suspected catheter-related infection (CRI) (new episode of fever or sepsis) were included. Cultures of insertion skin site, paired blood samples, catheter-tip, and other clinical samples were taken. Capability of insertion skin site culture to predict CRBSI and PBSI was determined.

Results We included 108 CVC from 96 CRI suspicion episodes. The causes that motivated CRI suspicion were 20 (18.5\%) PBSI, 44 (40.7\%) other infections, and 44 (40.7\%) unknown. Among the 20 PBSI, 11 (55\%) were CRBSI and 9 (45\%) were BSIUO. Negative predictive value of insertion skin site culture to predict CRBSI was 95\% (87-98\%) and to predict PBSI was $85 \%(76-91 \%)$.

Conclusions The new finding of our study was that skin insertion site culture had a good negative predicted valued for the prediction of CRBSI and PBSI.
\end{abstract}

Keywords Bloodstream infection $\cdot$ Prediction $\cdot$ Skin insertion site culture

\section{Introduction}

\section{Highlights}

- Skin insertion site culture for prediction of CRBSI and PBSI

- Skin insertion site culture had a good negative predicted valued for the prediction of CRBSI and PBSI

Leonardo Lorente

lorentemartin@msn.com

María Lecuona

mlecuona2005@yahoo.es

Alejandra Pérez-Llombet

alejandrapllombet@gmail.com

Adriana González-Mesa

adrianaglezmesa@gmail.com

Manuel Callejon

macafer4@gmail.com

Alejandro Jiménez

ajimenezsosa@gmail.com
Recent guidelines for the management of intravascular catheter-related infection (CRI) recommended that the routinely immediate removal of the central venous catheter

\section{María Luisa Mora}

mmorquic@gobiernodecanarias.org

Ana Madueño

ana_madueno@hotmail.com

1 Intensive Care Unit, Hospital Universitario de Canarias, La Laguna 38320, OfraTenerife, s/n, Spain

2 Microbiology and Infection Control Service, Hospital Universitario de Canarias, La Laguna 38320, OfraTenerife, $\mathrm{s} / \mathrm{n}$, Spain

3 Research Unit, Hospital Universitario de Canarias, La Laguna 38320, Ofra, s/nTenerife, Spain 
(CVC) is not necessary in patients who are hemodynamically stable, without immunosuppressive therapy, intravascular foreign bodies or organ transplantation, and no suppuration at the insertion site or bacteremia/fungemia [1-3].

There are several arguments against the routinely immediate CVC removal when CRI is suspected, such as that critically ill patients frequently develop fever due other causes [4], vascular catheterization by new puncture entails risk of serious mechanical complications [5], and the absence of differences in outcome between the early CVC removal and watchful waiting group when CRI is suspected $[6,7]$.

Therefore, the use of conservative methods for the diagnosis of CRI that allow keeping the catheter in situ can have the advantage of avoiding unnecessary removal of the catheter and the potential risk of mechanical complications [8]. The semiquantitative cultures of CVC insertion skin site is one of those conservative methods. Previous studies have analyzed the capability of skin insertion site culture to predict catheter-related bloodstream infection (CRBSI) or CVC tip colonization [9-16]. CRBSI has been defined in those studies as a positive blood culture by recognized pathogen, CVC tip colonization with the same microorganism, and no other apparent infection source. However, there has been not analyzed its capability to predict primary bloodstream infection (PBSI) that include CRBSI and bloodstream infection of unknown origin (BSIUO) [17]. Thus, the novel objective of our study was to determine the capability of insertion skin site culture to predict CRBSI and PBSI.

\section{Methods}

\section{Design and subjects}

We performed a prospective and observational study between June 2020 and January 2021 with the approval of the Institutional Ethic Review Board of the Hospital Universitario de Canarias (Tenerife, Spain). The requirement of written informed consent was waived due to the only change of our daily clinical practice by the study was the skin insertion site culture (which is a noninvasive procedure that is internationally accepted for CRBSI diagnosis in the daily clinical practice) and due to that the prohibition of patient visits by the public health outbreak policy of Spanish Government due to the context of COVID-19 pandemia.

We included patients admitted to Intensive Care Unit underwent to some CVC at least during 7 days and CRI was suspected. CRI was suspected when a patient developed a new episode of fever or sepsis. Fever was considered as temperature $\geq 38{ }^{\circ} \mathrm{C}$. Sepsis was defined according to Sepsis-3 Consensus criteria of 2016 [18].

\section{Variables recorded}

The following variables were recorded for each patient: age, sex, diagnosis on admission, diabetes mellitus, renal replacement therapy previously to admission, chronic obstructive pulmonary disease (COPD), asthma, chronic liver disease, smoking, parenteral nutrition previously to admission, corticosteroids previously to admission, immunosuppressive therapy previously to admission, hematological tumor, solid tumor, human immunodeficiency virus, corticosteroids at sepsis, immunosuppressive therapy at sepsis, parenteral nutrition at sepsis, propofol at sepsis, renal replacement therapy at sepsis, temperature, lactic acid, glucose, creatinine, protein, albumin, C-reactive protein, procalcitonin, leukocytes, neutrophils, platelets, international normalized ratio (INR), activated partial thromboplastin time (aPTT), pressure of arterial oxygen/ fraction inspired of oxygen $\left(\mathrm{PaO}_{2} / \mathrm{FIO}_{2}\right)$, Sepsis-related Organ Failure Assessment (SOFA) score [19], time of CVC, site of CVC, aspect of skin insertion site, culture of skin insertion site, death at 30 days, and time alive during first 30 days.

\section{Sample collections}

The following samples were collected from each patient: insertion skin site culture, paired blood samples, cathetertip, and other clinical samples. Superficial swab from insertion site of approximately $3-\mathrm{cm}$ area of skin was taken after dressing and rubbing the area around the insertion site with a cotton swab wet with sterile saline. Paired blood samples were taken from peripheral vein, with $10-\mathrm{ml}$ blood sample in each one and separated by $15 \mathrm{~min}$. Catheter-tip sample was taken after scrubbing the skin surrounding the insertion site with $2 \%$ chlorhexidine and cutting off the tip (distal 5-cm segment) using sterile scissors. Patients without blood culture, catheter tip culture, and skin insertion site cultures were excluded of the analysis.

\section{Definitions}

We used criteria of European Centre for Disease Prevention and Control (ECDC) to define infections [17]. Cathetertip colonization was considered as significant growth of a microorganism on the CVC tip ( $\geq 15$ colony-forming units) by semi-quantitative method described by Maki et al. [20]. Insertion skin site colonization was considered as significant growth of a microorganism on semi-quantitative culture $(\geq 15$ colony-forming units per plate). BSIUO was defined as bloodstream infection of unknown origin (verified during survey and no source found). PBSI include CRBSI and BSIUO; thus, some PBSI had positive CVC tip colonization and others not. 


\section{Statistical analysis}

Continuous variables are reported as means and standard deviations, and categorical variables as frequencies and percentages. We used Mann-Whitney T test to compare continuous variables between groups. Comparison of categorical variables between groups was performed using chi-square test. We obtained the sensitivity, specificity, positive predictive value, negative predictive value, positive likelihood ratio, and negative likelihood ratio of the insertion skin site culture to determine the capability of insertion skin site culture to predict CRBSI and PBSI. All values were calculated with a 95\% confidence interval. $\mathrm{P}<0.05$ were considered statistically significant. Statistical analysis was performed with SPSS 17.0 (SPSS Inc., Chicago, IL, USA).

\section{Results}

We included 108 CVC from 96 episodes of CRI suspicion. The causes of clinical symptoms that motivated CRI suspicion were the following: 20 (18.5\%) PBSI, 44 $(40.7 \%)$ secondary to other infections, and $44(40.7 \%)$ unknown origin. Among the 20 PBSI, 11 (55\%) were CRBSI and $9(45 \%)$ were BSIUO. The site of CVC was $25(23.1 \%)$ subclavian, $53(49.1 \%)$ jugular, and 30 (27.8\%) femoral.

We found that the group of CVC developing CRBSI $(n=11)$ compared to CVC group without it $(n=97)$ had more rate of positive culture of skin insertion site $(\mathrm{p}=0.001)$ and of female $(\mathrm{p}=0.03)$; however, no significant differences were found in rate of death $(\mathrm{p}=0.99)$, time of $\mathrm{CVC}$, site of CVC, aspect of skin insertion site, and in the other variables that were recorded (Table 1).

We found that the group of CVC developing PBSI $(n=20)$ compared to CVC group without it $(n=88)$ had more rate of positive culture of skin insertion site $(p=0.03)$; however, no significant differences were found in rate of death $(p=0.78)$, time of CVC, site of $\mathrm{CVC}$, aspect of skin insertion site, and in the other variables that were recorded (Table 2).

We found 15 cases of positive culture of skin insertion. We found that the culture of skin insertion site was positive in 6 of 11 cases of CRBSI and negative in 88 of 97 cases without CRBSI (Table 3). We found that the culture of skin insertion site was positive in 6 of 20 cases of PBSI and negative in 79 of 88 cases without PBSI (Table 4). Table 5 describes sensitivity, specificity, positive predictive value, negative predictive value, positive likelihood ratio, and negative likelihood ratio of insertion skin site culture to predict CRBSI and PBSI.

\section{Discussion}

Previous studies have been found that culture of skin insertion site had a good negative predicted valued for the prediction of the CRBSI or CVC tip colonization [9-16]. Novel aspects of our research were that we studied the capability of skin insertion site culture for predict CRBSI and PBSI. The new finding of our study was that skin insertion site culture had a good negative predicted valued for the prediction of CRBSI and PBSI. Thus, that the probability of not having PBSI if the result of skin insertion site culture is negative is high.

Previous studies analyzed CRBSI defined as a positive blood culture by recognized pathogen (or two positive blood cultures by skin contaminant microorganism) obtained from a peripheral vein and CVC tip colonization with the same microorganism. However, we analyzed PBSI for the first time. The studies that have analyzed the capability prognosis of skin insertion site culture have many differences between them. Some studies included all patients removing CVC $[11,15,16]$, and other studies include only patients who CVC were removed due to catheter related infection suspicion $[9,10,12-14]$. Some studies determined the ability of skin insertion site culture to predict CVC colonization tip [9, 11], and other studies to predict CRBSI [10, 12-16]. Some studies did not include femoral CVC [14-16], and only one study reported an important rate of femoral CVC removed (26\%) [13]. Some studies included CVC with any time of catheterization [13], other studies only included CVC with more than $48 \mathrm{~h}$ of catheterization [12,14], and other studies only included CVC with more than 7 days of catheterization $[10,15,16]$.

In the most of previous studies was analyzed the culture of skin insertion site and hubs together, and culture only of skin insertion site was analyzed only in a study [16]. However, in the study by the team of Bouza et al. were included all patients removing $\mathrm{CVC}$ and only $24 \%$ were removed due to CRI suspicion [16]. Besides, in the study by Bouza et al., femoral CVC was not reported [16], and this may be a point of interest due to femoral site is associated with higher CRBSI risk [21].

Recent guidelines for the management of CRI recommended that immediate CVC removal is not necessary routinely in patients who are hemodynamically stable, without immunosuppressive therapy, intravascular foreign bodies or organ transplantation, and no suppuration at the insertion site or bacteremia/fungemia [1-3]. If any of these conditions is present, the strategy of watchful waiting and maintain CVC to microbiological results could be adopted. In addition, we believe that in the decision of watchful waiting or immediate CVC removal in patients with suspected CRI should take into account the vascular 
Table 1 Characteristics of CVC developing and not catheter-related bloodstream infection (CRBSI)

\begin{tabular}{|c|c|c|c|}
\hline Data & Non CRBSI $(n=97)$ & CRBSI $(\mathrm{n}=11)$ & P-value \\
\hline Culture positive of skin insertion site, $\mathrm{n}(\%)$ & $9(9.3)$ & $6(54.5)$ & 0.001 \\
\hline Aspect of skin insertion site, $\mathrm{n}(\%)$ & & & 0.34 \\
\hline Normal & $57(58.8)$ & $8(72.7)$ & \\
\hline Inflammation & $34(35.1)$ & $2(18.2)$ & \\
\hline Non-purulent exudate & $4(4.1)$ & 0 & \\
\hline Purulent exudate & $2(2.1)$ & $1(9.1)$ & \\
\hline Time of CVC (days)-median (p 25-75) & $9(7-12)$ & $9(8-12)$ & 0.41 \\
\hline Site of CVC, $\mathrm{n}(\%)$ & & & 0.66 \\
\hline Subclavian & $22(22.7)$ & $3(27.3)$ & \\
\hline Jugular & $49(50.5)$ & $4(36.4)$ & \\
\hline Femoral & $26(26.8)$ & $4(36.4)$ & \\
\hline Age, years (p 25-75) & $65(56-72)$ & $63(52-70)$ & 0.37 \\
\hline Sex female, $\mathrm{n}(\%)$ & $30(30.9)$ & 0 & 0.03 \\
\hline Admission diagnostic, $\mathrm{n}(\%)$ & & & 0.17 \\
\hline Medical & $72(74.2)$ & $9(81.8)$ & \\
\hline Surgical & $18(18.6)$ & 0 & \\
\hline Traumatology & $7(7.2)$ & $2(18.2)$ & \\
\hline Diabetes mellitus, $\mathrm{n}(\%)$ & $30(30.9)$ & $4(36.4)$ & 0.74 \\
\hline Renal replacement therapy previously to admission, $\mathrm{n}(\%)$ & $3(3.1)$ & $1(9.1)$ & 0.35 \\
\hline COPD, n (\%) & $14(14.4)$ & 0 & 0.35 \\
\hline Asthma, n (\%) & $5(5.2)$ & $1(9.1)$ & 0.48 \\
\hline Chronic liver disease, n (\%) & $4(4.1)$ & 0 & 0.99 \\
\hline Smoking, $\mathrm{n}(\%)$ & $15(15.5)$ & $1(9.1)$ & 0.99 \\
\hline Parenteral nutrition previously to admission, $\mathrm{n}(\%)$ & $2(2.1)$ & 0 & 0.99 \\
\hline Corticosteroids previously to admission, $\mathrm{n}(\%)$ & $5(5.2)$ & 0 & 0.99 \\
\hline Immunosuppressive therapy previously to admission, $\mathrm{n}(\%)$ & $5(5.2)$ & $1(9.1)$ & 0.48 \\
\hline Hematological tumor, n (\%) & 0 & $1(9.1)$ & 0.10 \\
\hline Solid tumor, $\mathrm{n}(\%)$ & $1(1.0)$ & 0 & 0.99 \\
\hline Human Immunodeficiency Virus, $\mathrm{n}(\%)$ & $1(1.0)$ & 0 & 0.99 \\
\hline Corticosteroids at sepsis, n (\%) & $16(16.5)$ & $1(9.1)$ & 0.99 \\
\hline Immunosuppressive therapy at sepsis, $\mathrm{n}(\%)$ & $3(3.1)$ & 0 & 0.99 \\
\hline Parenteral nutrition at sepsis, $\mathrm{n}(\%)$ & $16(16.5)$ & $2(18.2)$ & 0.99 \\
\hline Propofol at sepsis, n (\%) & $37(38.1)$ & $4(36.4)$ & 0.99 \\
\hline Renal replacement therapy at sepsis, $n(\%)$ & $13(13.4)$ & $1(9.1)$ & 0.99 \\
\hline Temperature, median, ${ }^{\circ} \mathrm{C}(\mathrm{p} 25-75)$ & $37.4(36.4-37.8)$ & $37.0(35.5-37.5)$ & 0.15 \\
\hline Lactic acid, median mmol/L (p 25-75) & $0.9(0.8-1.5)$ & $1.5(0.8-1.5)$ & 0.31 \\
\hline Glucose (g/dL)-median (p 25-75) & $125(102-148)$ & $114(103-142)$ & 0.67 \\
\hline Creatinine (mg/dl)-median (p 25-75) & $0.8(0.5-1.4)$ & $0.7(0.6-0.9)$ & 0.93 \\
\hline Protein (g/L), median (p 25-75) & $5.7(5.3-6.1)$ & $5.8(5.0-6.0)$ & 0.61 \\
\hline Albumin (g/L), median (p 25-75) & $2.8(2.7-3.3)$ & $2.8(2.6-2.8)$ & 0.87 \\
\hline C-reactive protein $(\mathrm{mg} / \mathrm{gl})$, median $(\mathrm{p} 25-75)$ & $95(38-151)$ & $94(24-169)$ & 0.90 \\
\hline Procalcitonin (ng/ml) - median (p 25-75) & $0.3(0.1-0.7)$ & $0.1(0.1-0.3)$ & 0.21 \\
\hline Leukocytes, median $\times 10^{3} / \mathrm{mm}^{3}(\mathrm{p} 25-75)$ & $11.4(8.4-15.0)$ & $11.0(8.0-12.2)$ & 0.45 \\
\hline Neutrophils, median $\times 10^{3} / \mathrm{mm}^{3}(\mathrm{p} 25-75)$ & $9.0(6.3-12.0)$ & $7.9(5.7-9.9)$ & 0.46 \\
\hline Platelets, median $\times 10^{3} / \mathrm{mm}^{3}(\mathrm{p} 25-75)$ & $246(183-334)$ & $254(146-335)$ & 0.68 \\
\hline INR, median (p 25-75) & $1.1(1.0-1.2)$ & $1.1(1.0-1.2)$ & 0.58 \\
\hline aPTT, median seconds (p 25-75) & $30(27-33)$ & $29(22-33)$ & 0.81 \\
\hline $\mathrm{PaO}_{2} / \mathrm{FIO}_{2}$ ratio, median (p 25-75) & $293(217-316)$ & $284(202-346)$ & 0.74 \\
\hline SOFA score, median (p 25-75) & $4(3-6)$ & $4(2-6)$ & 0.66 \\
\hline Deaths at 30 days, no. $(\%)$ & $26(26.8)$ & $3(27.3)$ & 0.99 \\
\hline Time alive during first 30 days (days), median ( $\mathrm{p} 25-75$ ) & $30(23-30)$ & $30(6-30)$ & 0.79 \\
\hline
\end{tabular}

$C V C$ central venous catheter, $C O P D$ chronic obstructive pulmonary disease, INR international normalized ratio, $a P T T$ activated partial thromboplastin time, $\mathrm{PaO}_{2} / \mathrm{FIO}_{2}$ pressure of arterial oxygen/fraction inspired oxygen, SOFA Sepsis-related Organ Failure Assessment 
Table 2 Characteristics of CVC developing and not primary bloodstream infections (PBSI)

\begin{tabular}{|c|c|c|c|}
\hline Data & Non PBSI $(\mathrm{n}=88)$ & PBSI $(n=20)$ & P-value \\
\hline Culture positive of skin site, $\mathrm{n}(\%)$ & $9(10.2)$ & $6(30.0)$ & 0.03 \\
\hline Aspect of skin insertion site, $\mathrm{n}(\%)$ & & & 0.31 \\
\hline Normal & $50(56.8)$ & $15(75.0)$ & \\
\hline Inflammation & $32(36.4)$ & $4(20.0)$ & \\
\hline Non-purulent exudate & $4(4.5)$ & 0 & \\
\hline Purulent exudate & $2(2.3)$ & $1(5.0)$ & \\
\hline Time of CVC (days), median ( $\mathrm{p} 25-75$ ) & $9(7-12)$ & $10(8-12)$ & 0.60 \\
\hline Site of CVC, $\mathrm{n}(\%)$ & & & 0.84 \\
\hline Subclavian & $21(23.9)$ & $4(20.0)$ & \\
\hline Jugular & $42(47.7)$ & $11(55.5)$ & \\
\hline Femoral & $25(28.4)$ & $5(25.0)$ & \\
\hline Age, years (p 25-75) & $65(56-72)$ & $64(54-72)$ & 0.65 \\
\hline Sex female, $\mathrm{n}(\%)$ & $28(31.8)$ & $2(10.0)$ & 0.06 \\
\hline Admission diagnostic, $\mathrm{n}(\%)$ & & & 0.09 \\
\hline Medical & 63 (71.6) & $18(90.0)$ & \\
\hline Surgical & $18(20.5)$ & 0 & \\
\hline Traumatology & $7(8.0)$ & $2(10.0)$ & \\
\hline Diabetes mellitus, $\mathrm{n}(\%)$ & $30(34.1)$ & $4(20.0)$ & 0.29 \\
\hline Renal replacement therapy previously to admission, $\mathrm{n}(\%)$ & $3(3.4)$ & $1(5.0)$ & 0.57 \\
\hline COPD, n $(\%)$ & $12(13.6)$ & $2(10.0)$ & 0.99 \\
\hline Asthma, n (\%) & $5(5.7)$ & $1(5.0)$ & 0.99 \\
\hline Chronic liver disease, $\mathrm{n}(\%)$ & $4(4.5)$ & 0 & 0.99 \\
\hline Smoking, $\mathrm{n}(\%)$ & $14(15.9)$ & $2(10.0)$ & 0.73 \\
\hline Parenteral nutrition previously to admission, $\mathrm{n}(\%)$ & $2(2.3)$ & 0 & 0.99 \\
\hline Corticosteroids previously to admission, $\mathrm{n}(\%)$ & $5(5.7)$ & 0 & 0.58 \\
\hline Immunosuppressive therapy previously to admission, $\mathrm{n}(\%)$ & $5(5.7)$ & $1(5.0)$ & 0.99 \\
\hline Hematological tumor, n (\%) & 0 & $1(5.0)$ & 0.19 \\
\hline Solid tumor, $\mathrm{n}(\%)$ & $1(1.1)$ & 0 & 0.99 \\
\hline Human Immunodeficiency Virus, $\mathrm{n}(\%)$ & $1(1.1)$ & 0 & 0.99 \\
\hline Corticosteroids at sepsis, n (\%) & $13(14.8)$ & $4(20.0)$ & 0.52 \\
\hline Immunosuppressive therapy at sepsis, $\mathrm{n}(\%)$ & $3(3.4)$ & 0 & 0.99 \\
\hline Parenteral nutrition at sepsis, n (\%) & $13(14.8)$ & $5(25.0)$ & 0.32 \\
\hline Propofol at sepsis, n (\%) & $35(39.8)$ & $6(30.0)$ & 0.46 \\
\hline Renal replacement therapy at sepsis, $n(\%)$ & $13(14.8)$ & $1(5.0)$ & 0.46 \\
\hline Temperature, median, ${ }^{\circ} \mathrm{C}(\mathrm{p} 25-75)$ & $37.4(36.6-37.8)$ & $37.2(35.0-38.0)$ & 0.52 \\
\hline Lactic acid, median mmol/L (p 25-75) & $0.9(0.8-1.4)$ & $1.2(0.8-1.5)$ & 0.35 \\
\hline Glucose (g/dL), median (p 25-75) & $125(102-149)$ & $126(106-145)$ & 0.83 \\
\hline Creatinine (mg/dl), median (p 25-75) & $0.8(0.5-1.5)$ & $0.7(0.6-0.9)$ & 0.37 \\
\hline Protein (g/L), median (p 25-75) & $5.7(5.3-6.1)$ & $5.9(5.0-6.0)$ & 0.82 \\
\hline Albumin (g/L), median (p 25-75) & $2.8(2.7-3.3)$ & $2.8(2.6-2.8)$ & 0.41 \\
\hline C-reactive protein $(\mathrm{mg} / \mathrm{gl})$, median $(\mathrm{p} 25-75)$ & $98(38-143)$ & $86(31-168)$ & 0.83 \\
\hline Procalcitonin (ng/ml), median (p 25-75) & $0.3(0.1-0.8)$ & $0.1(0.1-0.4)$ & 0.37 \\
\hline Leukocytes, median $\times 10^{3} / \mathrm{mm}^{3}(\mathrm{p} 25-75)$ & $11.5(8.3-14.7)$ & $11.0(8.3-13.4)$ & 0.80 \\
\hline Neutrophils, median $\times 10^{3} / \mathrm{mm}^{3}(\mathrm{p} 25-75)$ & $8.5(6.3-12.0)$ & $8.9(5.8-12.0)$ & 0.99 \\
\hline Platelets, median $\times 10^{3} / \mathrm{mm}^{3}(\mathrm{p} 25-75)$ & $246(180-334)$ & $261(200-325)$ & 0.87 \\
\hline INR, median (p 25-75) & $1.1(1.0-1.2)$ & $1.1(1.0-1.2)$ & 0.83 \\
\hline aPTT, median seconds ( $\mathrm{p} 25-75$ ) & $30(27-33)$ & $29(26-33)$ & 0.97 \\
\hline $\mathrm{PaO}_{2} / \mathrm{FIO}_{2}$ ratio, median (p 25-75) & $293(229-335)$ & $253(200-300)$ & 0.33 \\
\hline SOFA score, median (p 25-75) & $4(3-6)$ & $5(2-7)$ & 0.91 \\
\hline Deaths at 30 days, no. (\%) & $23(26.1)$ & $6(30.0)$ & 0.78 \\
\hline Time alive during first 30 days (days), median ( $\mathrm{p} 25-75$ ) & $30(22-30)$ & $30(21-30)$ & 0.72 \\
\hline
\end{tabular}

$C V C$ central venous catheter, $C O P D$ chronic obstructive pulmonary disease, INR international normalized ratio, $a P T T$ activated partial thromboplastin time, $\mathrm{PaO}_{2} / \mathrm{FIO}_{2}$ pressure of arterial oxygen/fraction inspired oxygen, SOFA Sepsis-related Organ Failure Assessment 
Table 3 Test results of insertion skin site culture and existence of catheter-related bloodstream infection (CRBSI)

\begin{tabular}{llll}
\hline & CRBSI $(\mathrm{n}=11)$ & Non CRBSI $(\mathrm{n}=97)$ & Total $(\mathrm{n}=108)$ \\
\hline $\begin{array}{l}\text { Positive } \\
\text { insertion }\end{array}$ & $6(54.5 \%)$ & $9(9.3 \%)$ & $15(13.9 \%)$ \\
$\begin{array}{l}\text { skin site } \\
\text { culture }\end{array}$ & & & \\
$\begin{array}{l}\text { Negative } \\
\text { insertion } \\
\text { skin site } \\
\text { culture }\end{array}$ & $5(45.5 \%)$ & $88(90.7 \%)$ & $93(86.1 \%)$ \\
\hline
\end{tabular}

Table 4 Test results of insertion skin site culture and existence of primary bloodstream infections (PBSI)

\begin{tabular}{llll}
\hline & PBSI $(\mathrm{n}=20)$ & Non PBSI $(\mathrm{n}=88)$ & Total $(\mathrm{n}=108)$ \\
\hline $\begin{array}{l}\text { Positive } \\
\text { insertion } \\
\text { skin site } \\
\text { culture }\end{array}$ & $6(30.0 \%)$ & $9(10.2 \%)$ & $15(13.9 \%)$ \\
$\begin{array}{l}\text { Negative } \\
\text { insertion } \\
\text { skin site } \\
\text { culture }\end{array}$ & $14(70.0 \%)$ & $79(89.8 \%)$ & $93(86.1 \%)$ \\
\hline
\end{tabular}

accessibility (since that in some cases, new vascular catheterization may be very difficult due to poor vascular access) and the risk of mechanical complications (since that in same cases, as severe coagulopathy or respiratory disease could appears life-threatening complications). Thus, the development of methods for the diagnosis of CRBSI without CVC removal can contribute to unnecessary CVC removal and to reduce mechanical complications due to CVC. We think that the approach of skin insertion site culture could help in the decision of watchful waiting avoiding routinely immediate CVC removal in patients with suspected CRI.

Some limitations must be recognized in our study. First, we have not taken other non-invasive cultures to compare its capability to predict PBSI with skin insertion site culture. Second, we have not registered all CVC to know the incidence of PBSI and the rate of skin insertion site culture positive in all CVC. Third, we have not reported what proportion of CVC was excluded due to have not all culture (blood, catheter tip, and skin insertion site culture).

\section{Conclusion}

The new finding of our study was that skin insertion site culture had a good negative predicted valued for the prediction of CRBSI and PBSI.

Abbreviations aPTT: Activated partial thromboplastin time; CRBSI: Catheter-related bloodstream infection; CRI: Catheter-related infection; CVC: Central venous catheter; COPD: Chronic obstructive pulmonary disease; $\mathrm{FIO}_{2}$ : Fraction inspired of oxygen; INR: International normalized ratio; $\mathrm{PaO}_{2}$ : Pressure arterial of oxygen; PBSI: Primary bloodstream infection; SOFA: Sepsis-related Organ Failure Assessment score

Author contribution LL conceived, designed, and coordinated the study; participated in acquisition and interpretation of data; and drafted the manuscript. ML and AM conceived and designed the study and participated in acquisition and interpretation of data. APL, AGM, MC, and MLM participated in acquisition of data. AJ participated in the interpretation of data. All authors revised the manuscript critically for important intellectual content, made the final approval of the version to be published, and were agreed to be accountable for all aspects of the work.

Funding This study was supported by a grant from Fundación DISA a la Investigación Médica 2019 (Santa Cruz de Tenerife. Spain) and a grant from Instituto de Salud Carlos III (PI-18-00500) (Madrid, Spain) and co-financed with Fondo Europeo de Desarrollo Regional (FEDER).

Availability of data and material The datasets used and/or analyzed during the current study are available from the corresponding author on reasonable request.

\section{Declarations}

Ethics approval This study was carried out with the approval of the Institutional Ethic Review Board of the Hospital Universitario de Canarias (Tenerife, Spain). The requirement of written informed consent was waived due to the only change of our daily clinical practice by the study was the skin insertion site culture (which is a noninvasive procedure that is internationally accepted for CRBSI diagnosis in the daily clinical practice) and due to that the prohibition of patient visits
Table 5 Capacity of insertion skin site culture to predict catheter-related bloodstream infection (CRBSI) and primary bloodstream infections (PBSI)

\begin{tabular}{lcr}
\hline & \multicolumn{1}{l}{ CRBSI } & \multicolumn{1}{c}{ PBSI } \\
\hline Sensitivity and 95\% CI & $55 \%(25-82 \%)$ & $30 \%(13-54 \%)$ \\
Specificity and 95\% CI & $91 \%(83-95 \%)$ & $90 \%(81-95 \%)$ \\
Positive predicted value and 95\% CI & $40 \%(17-67 \%)$ & $40 \%(17-67 \%)$ \\
Negative predicted value and 95\% CI & $95 \%(87-98 \%)$ & $85 \%(76-91 \%)$ \\
Positive likelihood ratio and 95\% CI & $5.88(2.58-13.40)$ & $2.93(1.18-7.30)$ \\
Negative likelihood ratio and 95\% CI & $0.50(0.26-0.96)$ & $0.78(0.58-1.05)$ \\
\hline
\end{tabular}

$C I$ confidence intervals 
by the public health outbreak policy of Spanish Government due to the context of COVID-19 pandemia.

Disclaimer The funders had no role in study design, data collection and analysis, decision to publish, or preparation of the manuscript.

Conflict of interest The authors declare no competing interests.

\section{References}

1. Timsit JF, Baleine J, Bernard L et al (2020) Expert consensusbased clinical practice guidelines management of intravascular catheters in the intensive care unit. Ann Intensive Care 10:118

2. Farina J, Cornistein W, Balasini C et al (2019) Infecciones asociadas a catéteres venosos centrales. Actualización y recomendaciones intersociedades [Central venous catheter related infections. Inter-Societies update and recommendations]. Medicina (B Aires) 79:53-60

3. Chaves F, Garnacho-Montero J, Del Pozo JL et al (2018) Diagnosis and treatment of catheter-related bloodstream infection: clinical guidelines of the Spanish Society of Infectious Diseases and Clinical Microbiology and (SEIMC) and the Spanish Society of Spanish Society of Intensive and Critical Care Medicine and Coronary Units (SEMICYUC). Med Intensiva 42:5-36

4. O'Grady NP, Barie PS, Bartlett JG et al (2008) American College of Critical Care Medicine; Infectious Diseases Society of America: guidelines for evaluation of new fever in critically ill adult patients: 2008 update from the American College of Critical Care Medicine and the Infectious Diseases Society of America. Crit Care Med 36:1330-1349

5. Lorente L, León C (2009) Femoral venous catheterization. Does it really need to be avoided?. Med Intensiva 33:442-449

6. Rijnders BJ, Peetermans WE, Verwaest C et al (2004) Watchful waiting versus immediate catheter removal in ICU patients with suspected catheter-related infection: a randomized trial. Intensive Care Med 30:1073-1080

7. Lorente L, Martín MM, Vidal P et al (2014) Working Group on Catheter Related Infection Suspicion Management of GTEIS/ SEMICYUC. Should central venous catheter be systematically removed in patients with suspected catheter related infection? Crit Care 18:564

8. Lorente L (2012) Conservative methods for diagnosing catheterassociated bacteremia. Med Intensiva 36:163-168

9. Cercenado E, Ena J, Rodríguez-Créixems M et al (1990) A conservative procedure for the diagnosis of catheter-related infections. Arch Intern Med 150:1417-1420
10. León M, García M, Herranz MA et al (1998) Diagnostic value of Gram staining of peri-catheter skin and the connection in the prediction of intravascular-catheter-related bacteremia. Enferm Infecc Microbiol Clin 16:214-218

11. Fortún J, Perez-Molina JA, Asensio A et al (2000) Semiquantitative culture of subcutaneous segment for conservative diagnosis of intravascular catheter-related infection. JPEN J Parenter Enteral Nutr 24:210-214

12. Bouza E, Alvarado N, Alcalá L et al (2007) A randomized and prospective study of 3 procedures for the diagnosis of catheterrelated bloodstream infection without catheter withdrawal. Clin Infect Dis 44:820-826

13. Gowardman JR, Jeffries P, Lassig-Smith M et al (2013) A comparative assessment of two conservative methods for the diagnosis of catheter-related infection in critically ill patients. Intensive Care Med 39:109-116

14. Kumar A, Sharma RM, Jaideep CN, Hazra N (2014) Diagnosis of central venous catheter-related bloodstream infection without catheter removal: a prospective observational study. Med J Armed Forces India 70:17-21

15. Pérez-Granda MJ, Guembe M, Cruces R, Bouza E (2016) Vascular catheter colonization: surveillance based on culture of needleless connectors. Crit Care 20:166

16. Pérez-Granda MJ, Guembe M, Cruces R et al (2016) Assessment of central venous catheter colonization using surveillance culture of withdrawn connectors and insertion site skin. Crit Care 20:32

17. European Centre for Disease Prevention and Control (2012) Point prevalence survey of health care associated infections and antimicrobial use in European acute care hospitals-protocol version 4.3. Stockholm: ECDC. Available from: https://ecdc.europa.eu/sites/ portal/files/media/en/publications/Publications/0512-TED-PPSHAI-antimicrobial-use-protocol.pdf

18. Singer M, Deutschman CS, Seymour CW et al (2016) The Third International Consensus Definitions for Sepsis and Septic Shock (Sepsis-3). JAMA 315:801-810

19. Vincent JL, Moreno R, Takala J et al (1996) The Sepsis-related Organ Failure Assessment (SOFA) score to describe organ dysfunction/failure. Intensive Care Med 22:707-710

20. Maki DG, Weise CE, Sarafin HW (1977) A semiquantitative culture method for identifying intravenous catheter-related infection. N Engl J Med 296:1305-1309

21. Lorente L, Henry C, Martín MM et al (2005) Central venous catheter-related infection in a prospective and observational study of 2595 catheters. Crit Care 9:631-635 